\title{
Erratum to: Reports of the IAU Working Group on Cartographic Coordinates and Rotational Elements: 2006 \& 2009
}

\author{
B. A. Archinal - M. F. A'Hearn - A. Conrad - G. J. Consolmagno - R. Courtin • \\ T. Fukushima - D. Hestroffer · J. L. Hilton · G. A. Krasinsky • G. Neumann • \\ J. Oberst - P. K. Seidelmann - P. Stooke - D. J. Tholen - P. C. Thomas • \\ I. P. Williams
}

Published online: 27 July 2011

(C) Springer Science+Business Media B.V. (outside the USA) 2011

\begin{abstract}
The primary poles for (243) Ida and (134340) Pluto and its satellite (134340) Pluto : I Charon were redefined in the IAU Working Group on Cartographic Coordinates and Rotational Elements (WGCCRE) 2006 report (Seidelmann et al. in Celest Mech Dyn Astr 98:155, 2007), and 2009 report (Archinal et al. in Celest Mech Dyn Astr 109:101, 2011), respectively, to be consistent with the primary poles of similar Solar System bodies. However, the WGCCRE failed to take into account the effect of the redefinition of the poles on the values of the rotation angle $W$ at $\mathbf{J} 2000.0$. The revised relationships in Table 3 of Archinal et al. (2011) are
\end{abstract}

The online version of the original articles can be found under doi:10.1007/s10569-007-9072-y and doi:10.1007/s10569-010-9320-4.

G. A. Krasinsky deceased on March 17, 2011.

B. A. Archinal $(\varangle)$

U.S. Geological Survey, Flagstaff, AZ, USA

e-mail: barchinal@usgs.gov

M. F. A'Hearn

University of Maryland, College Park, MD, USA

A. Conrad

Max Planck Institute for Astronomy, Heidelberg, Germany

G. J. Consolmagno

Vatican Observatory, Vatican City, Vatican City State, Italy

R. Courtin

LESIA, Observatoire de Paris, CNRS, Paris, France

T. Fukushima

National Astronomical Observatory of Japan, Tokyo, Japan

D. Hestroffer

IMCCE, Observatoire de Paris, CNRS, Paris, France 


$$
\begin{aligned}
& W=274^{\circ} .05+1864^{\circ} .6280070 d \text { for }(243) \text { Ida, } \\
& W=302^{\circ} .695+56^{\circ} .3625225 d \text { for }(134340) \text { Pluto, and } \\
& W=122^{\circ} .695+56^{\circ} .3625225 d \text { for }(134340) \text { Pluto : I Charon }
\end{aligned}
$$

where $d$ is the time in TDB days from J2000.0 (JD2451545.0).

Keywords Cartographic coordinates $\cdot$ Rotation axes $\cdot$ Rotation periods $\cdot$ Rotation angles · Pluto $\cdot$ Charon $\cdot$ Ida

\section{Erratum to: Celest Mech Dyn Astr (2007) 98:155-180 \\ DOI 10.1007/s10569-007-9072-y and \\ Celest Mech Dyn Astr (2011) 109:101-135 \\ DOI 10.1007/s10569-010-9320-4}

The primary pole for Solar System bodies other than planets and their satellites is the pole about which the body appears to rotate in a counterclockwise direction. However, for planets, the primary pole is the one on the north side of the Solar System's invariable plane. The obliquities of the equators of (134340) Pluto and (134340) Pluto: I Charon to the orbit of their barycenter around the Sun are large enough that the poles they rotate about in a counterclockwise direction are on the south side of the invariable plane. Thus, the designation of Pluto as a dwarf planet required redefinition of the primary poles of Pluto and Charon to remain consistent with the definition of the primary pole. These redefinitions were made in Table 3 of the 2009 report of the IAU Working Group on Cartographic Coordinates and Rotational Elements (WGCCRE) (Archinal et al. 2011).

Similarly, the original (prior to 2003) definition of (243) Ida's primary pole-that used for planets and satellites - was the (north) pole it rotated about in a clockwise direction from

J. L. Hilton

U.S. Naval Observatory, Washington, DC, USA

G. A. Krasinsky

Institute for Applied Astronomy, St. Petersburg, Russia

G. Neumann

NASA Goddard Space Flight Center, Greenbelt, MD, USA

J. Oberst

DLR Berlin Aldershof, Berlin, Germany

P. K. Seidelmann

University of Virginia, Charlottesville, VA, USA

P. Stooke

University of Western Ontario, London, Canada

D. J. Tholen

University of Hawaii, Honolulu, HI, USA

P. C. Thomas

Cornell University, Ithaca, NY, USA

I. P. Williams

Queen Mary University of London, London, UK 
above. Thus, to be consistent with the definition for other minor planets, the redefinition of Ida's primary was made in the 2006 report of the WGCCRE (Seidelmann et al. 2007).

Changing the definition of the primary pole requires not only a change in the pole position, but a change in the rotation angle, $W$. This angle is measured in an easterly direction along the body's equator from the node $Q$ (Archinal et al. 2011) to the intersection of the body's prime meridian and its equator. Node $Q$ is defined as the node at the intersection of the body's equator and the equator of the ICRF whose right ascension is $90^{\circ}+\alpha_{0}$ where $\alpha_{0}$ is the right ascension of the primary pole.

Switching the primary pole of a body is not a rotation, but a reflection with the body's equator as the plane of reflection. Performing this reflection results in the following changes:

1. The node designated as $Q$ switches from its original position to the other intersection of the body's equator and the equator of the ICRF.

2. The easterly direction along the body's equator switches direction.

3. By item 2, the sign of the rate of change of $W$ changes, but its absolute rate is unaffected.

4. By items 1 and 2, the value of $W_{0}, W$ at the standard epoch, becomes its supplementary angle

$$
W_{0}(\text { new })=180^{\circ}-W_{0}(\text { old })
$$

Seidelmann et al. (2007) and Archinal et al. (2011) did change the sign of the rate of $W$, but failed to adjust the value of $W_{0}$. The corrected relations for Table 3 are

$$
\begin{aligned}
& W=274.05+1864^{\circ} .6280070 d \text { for Ida, } \\
& W=302^{\circ} .695+56^{\circ} .3625225 d \text { for Pluto, and } \\
& W=122^{\circ} .695+56^{\circ} .3625225 d \text { for Pluto : I Charon, }
\end{aligned}
$$

where $d$ is the time in TDB days from J2000.0 (JD2451545.0).

\section{References}

Archinal, B.A., A’Hearn, M.F., Bowell, E., Conrad, A., Consolmagno, G.J., Courtin, R., Fukushima, T., Hestroffer, D., Hilton, J.L., Krasinsky, G.A., Neumann, G., Oberst, J., Seidelmann, P.K., Stooke, P., Tholen, D.J., Thomas, P.C., Williams, I.P., Celest. Mech. Dyn. Astr. 109, 101 (2011). doi:10.1007/ s10569-010-9320-4

Seidelmann, P.K., Archinal, B.A., A’Hearn, M.F., Conrad, A., Consolmagno, G.J., Hestroffer, D., Hilton, J.L., Krasinsky, G.A., Neumann, G., Oberst, J., Stooke, P., Tedesco, E.F., Tholen, D.J., Thomas, P.C., Williams, I.P., Celest. Mech. Dyn. Astr. 98, 155 (2007). doi:10.1007/s10569-007-9072-y 